Check for updates

Cite this: J. Mater. Chem. A, 2020, 8 , 21339

DOI: 10.1039/d0ta90212h

rsc.li/materials-a

\section{Correction: Structural characterization of a polycrystalline epitaxially-fused colloidal quantum dot superlattice by electron tomography}

\author{
Xiaolei Chu, ${ }^{a}$ Hamed Heidari, ${ }^{a}$ Alex Abelson, ${ }^{\mathrm{b}}$ Davis Unruh, ${ }^{\mathrm{c}}$ Chase Hansen, ${ }^{\mathrm{c}}$ \\ Caroline Qian, ${ }^{d}$ Gergely Zimanyi, ${ }^{c}$ Matt Law*bde and Adam J. Moulé*a
}

Correction for 'Structural characterization of a polycrystalline epitaxially-fused colloidal quantum dot superlattice by electron tomography' by Xiaolei Chu et al., J. Mater. Chem. A, 2020, DOI: 10.1039/ d0ta06704k.

The authors regret the following minor errors in the published article:

In Section 2.1 (Materials), two chemicals, lead iodide and ethanol, were accidentally omitted from the list. The revised text should read:

"Lead oxide ( $\mathrm{PbO}, 99.999 \%)$, lead iodide $\left(\mathrm{PbI}_{2}, 99.9985 \%\right.$, purchased from Alfa Aesar), oleic acid (OA, technical grade, 90\%), diphenylphosphine (DPP, 98\%), 1-octadecene (ODE, 90\%), ethanol (99.5\%, anhydrous), ethylene glycol (EG, 99.8\%, anhydrous), acetonitrile (99.99\%, anhydrous), hexanes ( $\geq 99 \%$, anhydrous), toluene (99.8\%, anhydrous), (3-mercaptopropyl)trimethoxysilane (3-MPTMS, 95\%), and N,N-dimethylformamide (DMF, 99.8\%, anhydrous) were purchased from Sigma Aldrich and used as received."

In Section 2.9 (Mobility simulation), the text "where $\nu$ is a suitably chosen prefactor... final state of the hooping. ${ }^{59 "}$ should instead read as follows:

"where $\nu$ is an attempt frequency, chosen to be $10^{12} \mathrm{~s}^{-1}, g_{\mathrm{ij}}$ is the product of the initial density of states on $\mathrm{QD}_{\mathrm{i}}$ and the final density of states on $\mathrm{QD}_{\mathrm{j}}$, and $\beta_{\mathrm{ij}}$ is the tunneling amplitude evaluated using the $\mathrm{WKB}$ approximation as

$$
\beta_{\mathrm{ij}}=\exp \left(-2 \Delta x \sqrt{\frac{2 m^{*}\left(E_{\mathrm{vac}}-E_{\mathrm{ij}}\right)}{\hbar^{2}}}\right) .
$$

Here $\Delta x$ is the minimal surface separation of the QDs. $m^{*}$ is the effective mass of electrons in the tunneling medium, approximated as $0.05 m_{\mathrm{e}}$, the effective mass of electrons in bulk PbSe. $E_{\mathrm{vac}}$ is the vacuum energy level that is set to be zero as all other energy levels are defined relative to the vacuum. $E_{\mathrm{ij}}$ is the tunneling energy, taken to be the average of the initial and final states of the tunneling transition: $E_{\mathrm{ij}}=\frac{E_{\mathrm{i}}+E_{\mathrm{j}}}{2}$, where $E_{\mathrm{i}}$ and $E_{\mathrm{j}}$ are the energy levels of $\mathrm{QD}_{\mathrm{i}}$ and $\mathrm{QD}_{\mathrm{j}}{ }^{59,}$

Finally, in Section 3.1 (SL unit cell and disorder), the text "with an average QD spacing of $a$ along the chains and $\sqrt{2} a$ between the chains" should instead read: "with an average QD spacing of $a$ along the chains and approximately $\sqrt{2} a$ between the chains".

The Royal Society of Chemistry apologises for these errors and any consequent inconvenience to authors and readers.

\footnotetext{
a Department of Chemical Engineering, University of California, Davis, USA. E-mail: amoule@ucdavis.edu ${ }^{b}$ Department of Materials Science and Engineering, University of California, Irvine, CA 92697, USA. E-mail: matt.law@uci.edu ${ }^{c}$ Department of Physics, University of California, Davis, USA

${ }^{d}$ Department of Chemical and Biomolecular Engineering, University of California, Irvine, CA 92697, USA

${ }^{e}$ Department of Chemistry, University of California, Irvine, CA 92697, USA
} 